\title{
On Design of Homogeneous Feedback Controllers for Finite-Time Stabilization of Stochastic Systems
}

\author{
Kenta Hoshino* \\ Department of Electronics and Electrical Engineering, \\ Aoyama Gakuin University, Kanagawa, Japan \\ E-mail: hoshino@ee.aoyama.ac.jp \\ Yûki Nishimura \\ Division of Mechanical Engineering, Graduate School of Science and Engineering, \\ Kagoshima University, Kagoshima, Japan \\ E-mail: yunishi@mech.kagoshima-u.ac.jp
}

\begin{abstract}
In this paper, we investigate the design of controllers for the finite-time stabilization of stochastic systems. In the stochastic finite-time stabilization using controllers designed by previous design methods, the input signals often exhibit the chattering-like behavior. This paper presents another method for designing controllers in stochastic finite-time stabilization problems to avoid the chattering-like behavior. Moreover, we discuss the cause of the chattering-like behavior in the finite-time stabilization through numerical results.
\end{abstract}

\section{Introduction}

This paper deals with designing controllers in the finite-time stabilization problem of stochastic systems. In particular, the proposed approach aims to avoid the chattering-like behavior in input signals.

The finite-time stability and stabilization have been dealt with by many authors in the nonlinear control community. The finite-time stability means that a system is stable and exhibits the finite-time convergence. The analysis of the finite-time stability of deterministic systems has been shown in [1] (see also [2, 3, 4]). The analysis provides a foundation of the finite-time stabilization. Those results enable the synthesis of the finite-time stable control systems, which are shown in $[5,6,4,7,8]$.

Stochastic systems also can exhibit the finite-time stability. For stochastic systems, the study [9] investigates the analysis of the finite-time stability. Following the analysis, the studies $[10,11,12,13]$ deal with the finitetime stabilization problem. Those studies show the stabilization of systems such as triangular systems and are based on homogeneity or backstepping-like techniques. Those studies develop the design methods as extensions of those for deterministic systems. However, when the

\footnotetext{
${ }^{*} \mathrm{He}$ is now with the Department of Systems Science, Kyoto University, Yoshida-honmachi, Sakyo-ku, Kyoto 6068501, Japan hoshino@i.kyoto-u.ac.jp
}

design methods for deterministic systems are merely extended to stochastic systems, due to the stochastic noise, undesired behavior appears.

As to stochastic systems, the input signals often show the chattering-like behavior in the finite-time stabilization. This is caused by merely applying design methods developed for deterministic systems to stochastic systems. We note that the non-Lipschitz continuity is a necessary condition of the finite-time stability. Therefore, finite-time stabilizing controllers are given by nonLipschitz continuous functions, and the non-Lipschitz continuity brings the non-smoothness structures into closed-loop systems. Then, the non-Lipschitz continuity and the stochastic noise cause the chattering-like behavior. Therefore, we need to develop another design to avoid the chattering-like behavior under the existence of the stochastic noises.

This paper shows a design method of finite-time stabilizing controllers such that the chattering-like behavior of input signals can be avoided. Previous studies heavily depend on the backstepping-like techniques in the design of the finite-time stabilizing feedback controllers $[10,13]$. The backstepping-like techniques are difficult to avoid the structure of the closed-loop systems exhibiting the chattering-like behavior. Instead of the backstepping-like techniques, this paper employs a homogeneity-based method. The design in this paper is based on the results in [14].

The rest of this paper is organized as follows. The following section introduces a motivating example that shows the chattering-like behavior. Then, we review the stochastic finite-time stability and the homogeneity. The following section gives the problem statements of this study, and we show a design of finite-time stabilizing controllers. We subsequently show a numerical example of avoiding chattering-like behavior.

Notations. We use the following notations. $\mathbb{R}$ is the set of real numbers and $\mathbb{R}^{n}$ is the $n$-dimensional Euclidean space. With the signum function $\operatorname{sgn}: \mathbb{R} \rightarrow \mathbb{R}$ which takes the values $\operatorname{sgn}(x)=1,-1,0$ for $x>0$, $x<0$, and $x=0$, respectively, we define the function 
$\operatorname{sig}(x)^{\alpha}$ as $\operatorname{sig}(x)^{\alpha}=\operatorname{sgn}(x)|x|^{\alpha}$ with $\alpha \in \mathbb{R}$. Moreover, $\mathbb{R}_{+}=(0, \infty)$. We denote the sample space, the $\sigma$-field, and the probability measure as $\Omega, \mathcal{F}$, and $\mathbb{P}$, respectively. With the filtration of $\mathcal{F}$, denoted as $\left\{\mathcal{F}_{t}\right\}_{t \geq 0}$, we assume that the filtered probability space $\left\{\Omega, \mathcal{F},\left\{\mathcal{F}_{t}\right\}_{t \geq 0}, \mathbb{P}\right\}$ satisfies the following conditions; the probability space $\{\Omega, \mathcal{F}, \mathbb{P}\}$ is compete, $\left\{\mathcal{F}_{t}\right\}_{t \geq 0}$ is right continuous with respect to $t$, and $\mathcal{F}_{0}$ includes all the $\mathbb{P}$-null sets of $\mathcal{F}$.

\section{Motivating Example}

We here provide an example motivating to avoid the chattering-like behavior in the stochastic finite-time stabilization.

The following example is mathematical, however, the example gives a motivation of this study, which is to avoid the chattering-like behavior of input signals. The example is given by

$$
\begin{aligned}
& d x_{1}=x_{2} d t+0.1 \operatorname{sig}\left(x_{1}\right)^{7 / 8} d w, \\
& d x_{2}=u d t,
\end{aligned}
$$

where $x=\left(x_{1}, x_{2}\right) \in \mathbb{R}^{2}$ is the state, $u \in \mathbb{R}$ is the input, and $w$ is a one-dimensional standard Wiener process. For the system (1), we apply the feedback controller given as

$$
u=k_{1}(x):=-3 \operatorname{sig}\left(\operatorname{sig}\left(x_{1}\right)^{9 / 4}+\operatorname{sig}\left(x_{2}\right)^{3}\right)^{2 / 9},
$$

which is designed by using the results in [6] with minor modifications. The feedback controller (2) guarantees the finite-time stability in probability (1) (see Definition 2 below).

Fig. 1 shows a sample path of $x(t)$ of (1) with (2). Fig. 1 indicates that $x(t)$ converges to $x=0$ in finite time. Moreover, Fig. 2 shows a sample path of the input $u(t)$. It also shows the finite-time convergence to zero. However, the input signal exhibits the chattering-like behavior. In actual control problems, this will cause difficulties in the implementation of the finite-time stabilizing controllers. Therefore, we deal with a design of finite-time stabilizing controllers for avoiding the chattering-like behavior of the input signals.

\section{Mathematical Preliminaries}

\subsection{Finite-time Stability of Stochastic Systems}

This subsection reviews the finite-time stability of stochastic systems.

Given the state variable $x \in \mathbb{R}^{n}$, the coefficient functions $f: \mathbb{R}^{n} \rightarrow \mathbb{R}^{n}$ and $\sigma: \mathbb{R}^{n} \rightarrow \mathbb{R}^{n \times d}$, and a $d$ dimensional standard Wiener process $w$, we consider the system

$$
d x=f(x) d t+\sigma(x) d w, \quad x(0)=x_{0} \in \mathbb{R}^{n},
$$

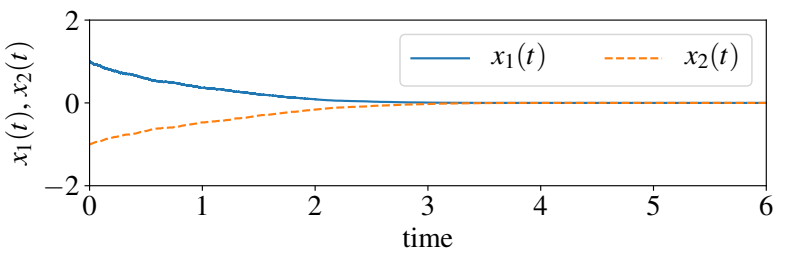

Fig. 1: Sample path of state $x(t)$ of system (1) with (2)

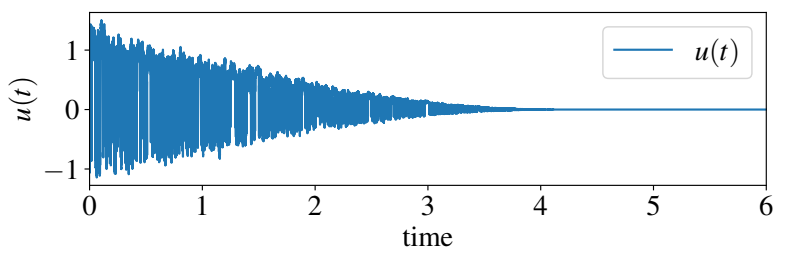

Fig. 2: Sample path of input $u(t)$ of system (1) with (2)

where $x_{0}$ is the initial value of the state. Throughout this subsection, we consider the finite-time stability of (3). Moreover, throughout this paper, we introduce the following assumptions; $f(x)$ and $\sigma(x)$ are continuous in $x$, and $f(0)=0$ and $\sigma(0)=0$. Because we do not necessarily require the Lipschitz continuity of the coefficients $f(x)$ and $\sigma(x)$ of the system (3), the solutions of (3) are defined as the weak solutions (for a detailed discussion of the definitions of solutions, see [15, Ch. $5]$ ). In the following, the solution of (3) is denoted as $x(t)$.

In the following, we discuss the stability of stochastic systems as in $[16,17]$.

Definition 1 (Stability in probability, [16, 17]). The origin $x=0$ of (3) is stable in probability if the following holds; for any $\epsilon>0$,

$$
\lim _{x_{0} \rightarrow 0} \mathbb{P}\left\{\sup _{t \geq 0}\|x(t)\|>\epsilon\right\}=0 .
$$

Then, we introduce the finite-time stability of stochastic systems as follows [9].

Definition 2 (Finite-time stability in probability, [9]). The origin $x=0$ of (3) is finite-time stable in probability if it is stable in probability and for a stopping time $\tau_{0}$ defined as

$$
\tau_{0}=\inf \{t \in(0, \infty) \mid\|x(t)\|=0\},
$$

it holds that for any $x_{0} \neq 0$,

$$
\mathbb{P}\left\{\tau_{0}<\infty\right\}=1 \text {. }
$$

The Lyapunov approach for finite-time stability has been established in [9]. For a twice continuously differentiable function $V: \mathbb{R}^{n} \rightarrow \mathbb{R}$, we introduce an operator given as

$\mathcal{L} V(x)=\frac{\partial V}{\partial x}(x) f(x)+\frac{1}{2} \operatorname{Tr}\left(\sigma(x)^{T} \frac{\partial}{\partial x}\left(\frac{\partial V}{\partial x}(x)\right)^{T} \sigma(x)\right)$. 
The operator $\mathcal{L}$ is called the infinitesimal generator of the system (3). Then, the following theorem allows us to investigate the finite-time stability of stochastic systems with Lyapunov functions, and this becomes a basis in the stochastic finite-time stabilization.

Theorem 1 ([9]). Let a function $V: \mathbb{R}^{n} \rightarrow \mathbb{R}$ be a twice continuously differentiable on $\mathbb{R}^{n}$. Moreover, assume that $V(x)$ is radially unbounded and positive definite on $\mathbb{R}^{n}$. For the system (3), if the inequality

$$
\mathcal{L} V(x) \leq-c V(x)^{\gamma}
$$

holds for some $c>0$ and $\gamma \in(0,1)$, then the origin of (3) is finite-time stable in probability.

\subsection{Homogeneity}

This subsection recalls the homogeneity of functions and vector fields (see [18, Chapter 5] for the details of the homogeneity). The homogeneity is employed in design methods of the finite-time stabilizing controllers in many studies $[5,6,7]$.

We begin with the definition of the dilation mapping.

Definition 3 (Dilation, [18]). Given parameters $r=$ $\left(r_{1}, \ldots, r_{n}\right) \in \mathbb{R}_{+}^{n}$ and $\lambda>0$, the mapping $\Delta_{\lambda}^{r}: \mathbb{R}^{n} \rightarrow$ $\mathbb{R}^{n}$ defined as $\Delta_{\lambda}^{r}(x)=\left(\lambda^{r_{1}} x_{1}, \ldots, \lambda^{r_{n}} x_{n}\right)$ is called the dilation mapping.

Then, with the dilation, we define the homogeneous functions and homogeneous vector fields.

Definition 4 (Homogeneous functions, [18]). Given parameters $r=\left(r_{1}, \ldots, r_{n}\right) \in \mathbb{R}_{+}^{n}, \lambda>0$, and a function $V: \mathbb{R}^{n} \rightarrow \mathbb{R}$, we call $V(x)$ a homogeneous function of degree $m$ with respect to the dilation $\Delta_{\lambda}^{r}$ if the function $V$ satisfies that $V\left(\Delta_{\lambda}^{r}(x)\right)=\lambda^{m} V(x)$.

Definition 5 (Homogeneous vector fields, [18]). Given parameters $r=\left(r_{1}, \ldots, r_{n}\right) \in \mathbb{R}_{+}^{n}, \lambda>0$, and a vector field $f: \mathbb{R}^{n} \rightarrow \mathbb{R}^{n}$, we call $f(x)$ a homogeneous vector field of degree $k$ with respect to a dilation $\Delta_{\lambda}^{r}$ if each $i$-th element $f_{i}(x)$ of the vector field $f(x)$ satisfies that $f_{i}\left(\Delta_{\lambda}^{r}(x)\right)=\lambda^{r_{i}+k} f_{i}(x)$.

For the later use, we define the homogeneous norm as follows.

Definition 6. Given $r=\left(r_{1}, \ldots, r_{n}\right)$ and sufficiently large $p>0$, we call $\|\cdot\|_{\{r, p\}}: \mathbb{R}^{n} \rightarrow \mathbb{R}$ the homogeneous norm if the mapping is given by $\|x\|_{\{r, p\}}=$ $\left(\sum_{i=1}^{n}\left|x_{i}\right|^{p / r_{i}}\right)^{1 / p}$

Then, we recall results related to the homogeneity.

Proposition 1 ([14]). Given two homogeneous functions $V_{1}, V_{2}: \mathbb{R}^{n} \rightarrow \mathbb{R}$ with respect to a dilation $\Delta_{\lambda}^{r}$ of degree $m_{1}$ and $m_{2}$, respectively, suppose that the function $V_{1}$ is positive definite. Then,

$$
k_{1} V_{1}(x)^{m_{2} / m_{1}} \leq V_{2}(x) \leq k_{2} V_{1}(x)^{m_{2} / m_{1}},
$$

where

$$
\begin{aligned}
& k_{1}=\min _{\left\{x \in \mathbb{R}^{n} \mid V_{1}(x)=1\right\}} V_{2}(x), \\
& k_{2}=\max _{\left\{x \in \mathbb{R}^{n} \mid V_{1}(x)=1\right\}} V_{2}(x) .
\end{aligned}
$$

Proposition 2 ([11]). Given a twice continuously differentiable function $V: \mathbb{R}^{n} \rightarrow \mathbb{R}$ and a vector field $f: \mathbb{R}^{n} \rightarrow \mathbb{R}^{n}$, suppose that the function $V(x)$ is homogeneous of degree $m$ with respect to a dilation $\Delta_{\lambda}^{r}$ and that the vector field $f(x)$ is homogeneous of degree $k$ with respect to the dilation $\Delta_{\lambda}^{r}$. Then, the following two functions are homogeneous of degree $m$ and $m+2 k$, respectively, with respect to the dilation $\Delta_{\lambda}^{r}$;

$$
\frac{\partial V}{\partial x}(x) f(x), \quad f(x)^{T} \frac{\partial}{\partial x}\left(\frac{\partial V}{\partial x}(x)\right)^{T} f(x) .
$$

\section{Problem Statements: Finite-Time Stabilization of Stochastic Systems}

This section gives the problem statements of this study. We deal with the finite-time stabilization of an $n$-th order integrator-like system.

Throughout this paper, given the state $x=$ $\left(x_{1}, x_{2}, \ldots, x_{n}\right) \in \mathbb{R}^{n}$ and the input $u \in \mathbb{R}$, we consider the stabilization of the $n$-th order integrator-like system given as

$$
\left(\begin{array}{c}
d x_{1} \\
d x_{2} \\
\vdots \\
d x_{n}
\end{array}\right)=\left(\begin{array}{c}
x_{2} \\
x_{3} \\
\vdots \\
u
\end{array}\right) d t+\Sigma(x) d w
$$

where $w$ is a one-dimensional standard Wiener process, and $\Sigma: \mathbb{R}^{n} \rightarrow \mathbb{R}^{n}$. We assume that the coefficient $\Sigma(x)$ is continuous in $x$ and $\Sigma(0)=0$.

The problem in this paper is primarily the finite-time stabilization of (5). That is, we deal with designing feedback controllers $u=k(x)$ such that the origin of (5) with the feedback controller $u=k(x)$ becomes finitetime stable in probability. In addition, we develop a design of finite-time stabilizing controllers to avoid the chattering-like behavior in the input signals, which we have seen in Section 2. Because previous design methods are based on the backstepping-like techniques and those methods causes the chattering-like behaviors, we show another approach to designing feedback controllers for the finite-time stabilization.

\section{Main Results: Design of Finite-Time Stabilizing Controllers}

This section describes a design of finite-time stabilizing controllers. The design is developed to avoid the 
chattering-like behavior in the input signals. The design is an extension of that for the finite-time stabilization of deterministic systems with the homogeneity [14], which differs from the backstepping-like techniques.

In the following theorem, we denote the set $S_{1}$ as

$$
S_{1}=\left\{x \in \mathbb{R}^{n} \mid\|x\|_{\{r, p\}}=1\right\},
$$

where the value of $r=\left(r_{1}, \ldots, r_{n}\right)$ is determined by a given dilation $\Delta_{\lambda}^{r}$ and $p>0$. The following result gives feedback controllers for the finite-time stabilization of stochastic systems, which is based on those for deterministic systems in [14].

Theorem 2. Consider the system (5) and a feedback controller given in the form of

$$
u=\kappa(x)=-k_{1} \operatorname{sig}\left(x_{1}\right)^{\alpha_{1}} \cdots-k_{n} \operatorname{sig}\left(x_{n}\right)^{\alpha_{n}},
$$

where the parameters $\alpha_{i}$ satisfy

$$
\alpha_{i-1}=\frac{\alpha_{i} \alpha_{i+1}}{2 \alpha_{i+1}-\alpha_{i}}
$$

for $i=2, \ldots, n, \alpha_{n}=\alpha$, and $\alpha_{n+1}=1$ for some $\alpha$. Further, assume that the vector field $\Sigma(x)$ is a homogeneous vector field of degree $k<0$ with respect to the dilation $\Delta_{\lambda}^{r}$ with

$$
r=\left(\frac{1}{\alpha_{1}}, \ldots, \frac{1}{\alpha_{n}}\right)
$$

for sufficiently small $|k|$ and satisfies that

$$
\|\Sigma(x)\|<\delta, \quad x \in S_{1}
$$

for sufficiently small $\delta>0$. Then, there exist the parameters $\alpha$ and $k_{i}(i=1, \ldots, n)$ such that the origin of the system (5) with the feedback controller (6) is finite-time stable in probability.

Proof. To show the finite-time stability, we first consider the deterministic system

$$
\left(\begin{array}{c}
\dot{x}_{1} \\
\dot{x}_{2} \\
\vdots \\
\dot{x}_{n}
\end{array}\right)=\bar{f}(x):=\left(\begin{array}{c}
x_{2} \\
x_{3} \\
\vdots \\
\kappa(x)
\end{array}\right),
$$

where $\kappa(x)$ is given by (6). The system (9) mathematically corresponds to the system (5) with the controller $(6)$ and $\Sigma(x) \equiv 0$. Then, according to Proposition 8.1 of [14], there exists $\epsilon>0$ such that the origin of (9) is finite-time stable with $\alpha \in(1-\epsilon, 1)$. Moreover, $\bar{f}(x)$ of $(9)$ is a homogeneous vector field of degree

$$
\bar{k}=\frac{\alpha-1}{\alpha}
$$

with respect to the dilation $\Delta_{\lambda}^{r}$ with the value of $r$ given in (7). Then, because of the homogeneity and the asymptotic stability of the system (9), according to
Theorem 2 of [18], there exists a function $\bar{V}: \mathbb{R}^{n} \rightarrow \mathbb{R}$ that is radially unbounded, positive definite, continuously differentiable of order $p$ on $\mathbb{R}^{n}$ for some $p$, and homogeneous with respect to the dilation $\Delta_{\lambda}^{r}$ and satisfies

$$
\dot{\bar{V}}(x):=\frac{\partial \bar{V}}{\partial x}(x) \bar{f}(x)<0 \text { for } \forall x \neq 0 .
$$

Because the function $\bar{V}(x)$ and the vector field $\bar{f}(x)$ are a homogeneous function and a homogeneous vector field, respectively, with respect to the dilation $\Delta_{\lambda}^{r}$, according to Proposition 2, the function $\dot{\bar{V}}$ is also a homogeneous function with respect to the dilation $\Delta_{\lambda}^{r}$.

Then, the closed-loop system (5) with the controller (6) is expressed as

$$
d x=\bar{f}(x) d t+\Sigma(x) d w,
$$

where $\bar{f}(x)$ is given in (9). When the value $|k|$ of the homogeneous degree $k<0$ of the vector field $\Sigma(x)$ with respect to the dilation $\Delta_{\lambda}^{r}$ is sufficiently small, we can choose the value of $\alpha$ in (10) so that $k=\bar{k} / 2$ holds. Then, with $k=\bar{k} / 2,(12)$ is a stochastic homogeneous system of degree $\bar{k}$ with respect to the dilation $\Delta_{\lambda}^{r}$. Then, for the function $\bar{V}(x)$, the infinitesimal generator of (12) results in

$$
\mathcal{L} \bar{V}(x)=\frac{\partial \bar{V}}{\partial x}(x) \bar{f}(x)+\frac{1}{2} \Sigma(x)^{T} \frac{\partial}{\partial x}\left(\frac{\partial \bar{V}}{\partial x}(x)\right)^{T} \Sigma(x) .
$$

Then, with $\dot{\bar{V}}(x)$ in $(11), \mathcal{L} \bar{V}(x)$ is rewritten as

$$
\mathcal{L} \bar{V}(x)=\dot{V}(x)+\frac{1}{2} \Sigma(x)^{T} \frac{\partial}{\partial x}\left(\frac{\partial \bar{V}}{\partial x}(x)\right)^{T} \Sigma(x) .
$$

Then, with Proposition 2, the first and second terms on the right-hand side of (13) are both homogeneous functions of the same homogeneous degrees with respect to the dilation $\Delta_{\lambda}^{r}$. Since the function $\dot{\bar{V}}(x)$ is negative definite, we consider the constant $\bar{\delta}>0$ defined as

$$
\bar{\delta}=\min _{x \in S_{1}}\{-\dot{\bar{V}}(x)\} .
$$

Then, with $\bar{\delta}$ of (14) and $\delta$ in the condition (8), for $x \in S_{1}$,

$$
\mathcal{L} \bar{V}(x) \leq-\bar{\delta}+\frac{1}{2} \delta^{2} \max _{x \in S_{1}}\left\{\left\|\frac{\partial}{\partial x}\left(\frac{\partial \bar{V}}{\partial x}(x)\right)^{T}\right\|\right\}
$$

holds. Then, the inequality (15) implies that for the sufficiently small value of $\delta$,

$$
\mathcal{L} \bar{V}(x)<0 \quad x \in S_{1}
$$

holds. Then, with the inequality (16) and the homogeneity of the function $\mathcal{L} \bar{V}(x)$, it is concluded that the function $\mathcal{L} \bar{V}(x)$ is negative definite by using Proposition 1. Moreover, Proposition 1 and the homogeneity 
of $\mathcal{L} \bar{V}(x)$ guarantee the existence of constants $c>0$ and $\gamma \in(0,1)$ such that

$$
\mathcal{L} \bar{V}(x) \leq-c \bar{V}(x)^{\gamma}
$$

holds. Finally, the inequality (17) implies the finitetime stability in probability of the closed-loop system (12).

Remark 1. The proof of Theorem 2 is based on the converse homogeneous Lyapunov theorem of [18]. Therefore, it does not provide the estimates of the values of $\delta$ in (8) and of $k$, which is the homogeneous degree of $\Sigma(x)$. In the numerical example shown in the next section, we provide the explicit form of the Lyapunov function $\bar{V}(x)$ for an example system and use the Lyapunov function to guarantee the stability.

\section{Numerical Example}

We show an example of the finite-time stabilization of stochastic systems with the design discussed in Section 5 .

In this section, we consider the continuation of the stabilization of the system (1) in Section 2. Based on the results in Section 5, we consider the feedback controller given by

$$
u=k_{2}(x):=-k_{1} \operatorname{sgn}\left(x_{1}\right)\left|x_{1}\right|^{1 / 2}-k_{2} \operatorname{sgn}\left(x_{2}\right)\left|x_{2}\right|^{2 / 3} .
$$

The feedback is obtained by setting $\alpha=2 / 3$ for (6). Moreover, with the value of $r$ given by

$$
r=\left(1 / \alpha_{1}, 1 / \alpha_{2}\right)=(2,3 / 2),
$$

the vector field $\Sigma(x)$ of the system (3) is a homogeneous vector field of degree $-1 / 4$ with respect to the dilation $\Delta_{\lambda}^{r}$. Then, we consider the function given by

$$
\bar{V}(x)=\frac{7}{4}\left|x_{1}\right|^{3}+\frac{1}{4} x_{2}^{4}+x_{2} \operatorname{sig}\left(x_{1}\right)^{\frac{9}{4}} .
$$

We note that $\bar{V}(x)$ of (19) is radially unbounded, positive definite, and twice continuously differentiable on $\mathbb{R}^{n}$. The function $\bar{V}(x)$ of (19) is a Lyapunov function indicating the finite-time stability of the closed-loop system. Indeed, a straightforward calculation shows that the infinitesimal generator of the closed-loop system (1) with the feedback controller (18) is negative definite on $\mathbb{R}^{2}$. In addition, by using the homogeneity, we can show the existence of $c>0$ and $\gamma \in(0,1)$ such that the condition (4) of the finite-time stability holds.

Figs. 3 and 4 show the sample paths of the state and the input, respectively, of the system (1) with the finitetime stabilizing controller (18) using the values $k_{1}=3$ and $k_{2}=3$, and the initial value of the state $x$ is given as $x(0)=(1,1)$. In Fig. $3, x(t)$ reaches $x=0$ in finite time. Moreover, the input signal $u(t)$ does not show the chattering-like behavior, which is different from the behavior of the controller (2) shown in Fig. 2. Therefore, the proposed design achieves the stabilization without the chattering-like behavior in the input signals.

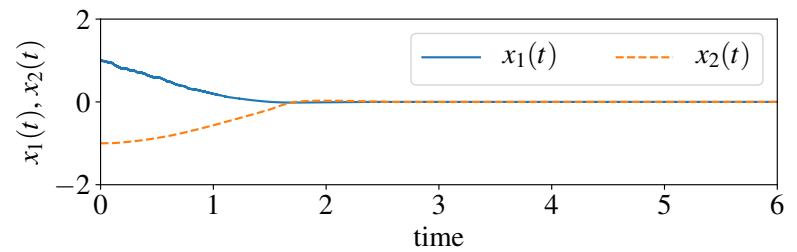

Fig. 3: Sample path of state $x(t)$ of system (1) with (18)

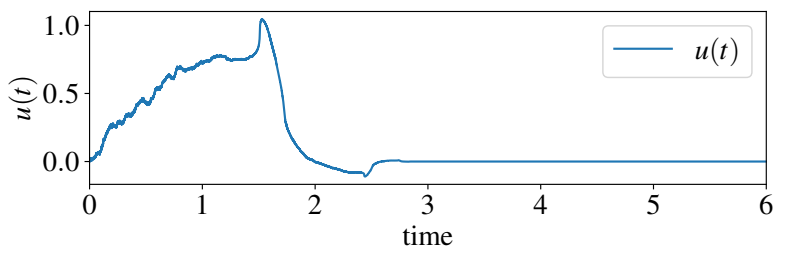

Fig. 4: Sample path of input $u(t)$ of system (1) with (18)

\section{Discussions}

Based on the results in Sections 5 and 6, this section discusses the difference between the finite-time stabilizing controller in Section 5 and the controllers designed by the backstepping-like methods, for example, the controller (2) in Section 2, in the view of the behavior of the input signals.

We examine the origin of the chattering-like behavior, which we have seen in Section 2. The feedback controller (2) is designed so that the state variable $x$ converges to the set given as

$$
S=\left\{\left.x \in \mathbb{R}^{2}\left|x_{2}=-\operatorname{sgn}\left(x_{1}\right)\right| x_{1}\right|^{3 / 4}\right\} .
$$

This can be shown in the plot of the function $k_{1}(x)$ of (2) (Fig. 5). In Fig. 5, the function $k_{1}(x)$ shows abrupt changes in the neighborhood of the set $S$ and this leads to the rapid convergence to the set $S$. In other words, the set $S$ plays similar roles to the sliding surfaces in the sliding-mode control. However, the state variable $x(t)$ crosses the set $S$ frequently because of the stochastic noise and this causes the chatteringlike behavior. On the other hand, we consider the function $k_{2}(x)$ of (18) which also gives controller (1), which guarantees the finite-time stability. Fig. 6 shows the plot of the function $k_{2}(x)$. The function $k_{2}(x)$ does not show abrupt changes unlike the function $k_{1}(x)$ shown in Fig. 5. This leads to avoiding the chattering-like behavior of the input signal with the finite-time stabilizing controller (18).

\section{Conclusions}

In this paper, we showed an approach for the finitetime stabilization of stochastic systems. In particular, we discussed the chattering-like behaviors in the input signals of finite-time stabilizing controllers designed by 


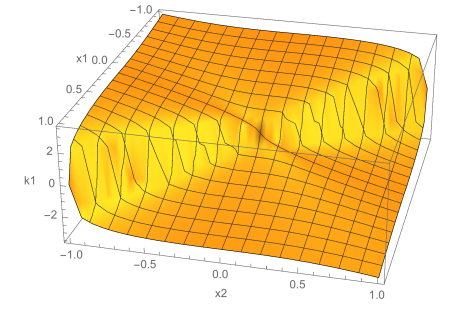

Fig. 5: Plot of function $k_{1}(x)$

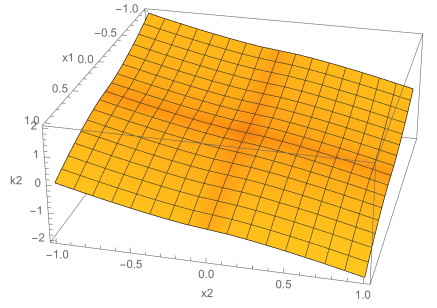

Fig. 6: Plot of function $k_{2}(x)$

backstepping-like methods and we considered another approach for designing the finite-time stabilizing controller for stochastic systems. The controllers shown in this study has a different form from the controller obtained by the backstepping-like methods. Through the numerical examples, we discussed that the controller obtained in this study could avoid the chattering-like behavior in the input signals.

We will deal with a constructive design of the finitetime stabilizing feedback controllers, which are shown in Theorem 2, in future works.

Acknowledgments: This work was partially supported by JSPS KAKENHI Grant Number JP15K18091 and JP17H03282.

\section{References}

[1] S. P. Bhat and D. S. Bernstein: Finite-time stability of continuous autonomous systems, SIAM Journal on Control and Optimization, vol. 38, no. 3, pp. 751-766, 2000.

[2] E. Roxin: On finite stability in control systems, Rendiconti del Circolo Matematico di Palermo, vol. 15, no. 3, pp. 273-282, 1966.

[3] V. T. Haimo: Finite time controllers, SIAM Journal on Control and Optimization, vol. 24, no. 4, pp. 760-770, 1986.

[4] E. Moulay and W. Perruquetti: Finite time stability and stabilization of a class of continuous systems, Journal of Mathematical analysis and applications, vol. 323, no. 2, pp. 1430-1443, 2006.

[5] S. P. Bhat and D. S. Bernstein: Continuous finitetime stabilization of the translational and rotational double integrators, IEEE Transactions on
Automatic Control, vol. 43, no. 5, pp. 678-682, 1998.

[6] Y. Hong: Finite-time stabilization and stabilizability of a class of controllable systems, Systems $\&$ control letters, vol. 46, no. 4, pp. 231-236, 2002.

[7] N. Nakamura, H. Nakamura, Y. Yamashita, and H. Nishitani: Homogeneous stabilization for input affine homogeneous systems, IEEE Transactions on Automatic Control, vol. 54, no. 9, pp. 2271$2275,2009$.

[8] A. Polyakov, D. Efimov, and W. Perruquetti: Finite-time and fixed-time stabilization: Implicit Lyapunov function approach, Automatica, vol. 51, pp. 332-340, 2015.

[9] J. Yin, S. Khoo, Z. Man, and X. Yu: Finite-time stability and instability of stochastic nonlinear systems, Automatica, vol. 47, no. 12, pp. 2671-2677, 2011.

[10] S. Khoo, J. Yin, Z. Man, and X. Yu: Finitetime stabilization of stochastic nonlinear systems in strict-feedback form, Automatica, vol. 49, no. 5, pp. 1403 - 1410, 2013.

[11] K. Hoshino, Y. Yamashita, Y. Nishimura, and D. Tsubakino: Stability and stabilization of homogeneous stochastic sytems, in Proceedings of the 52th IEEE Conference on Decision and Control, 2013, pp. 1229-1234.

[12] J. Yin, S. Khoo, and Z. Man: Finite-time stability theorems of homogeneous stochastic nonlinear systems, Systems \& Control Letters, vol. 100, pp. 6-13, 2017.

[13] F. Gao and Y. Wu: Global finite-time stabilisation for a class of stochastic high-order time-varying nonlinear systems, International Journal of Control, vol. 89, no. 12, pp. 2453-2465, 2016.

[14] S. P. Bhat and D. S. Bernstein: Geometric homogeneity with applications to finite-time stability, Mathematics of Control, Signals, and Systems (MCSS), vol. 17, no. 2, pp. 101-127, 2005.

[15] I. Karatzas and S. Shreve: Brownian motion and stochastic calculus, Springer, 2012, vol. 113.

[16] R. Khasminskii: Stochastic stability of differential equations, 2nd ed, Springer-Verlag Berlin Heidelberg, 2012.

[17] X. Mao: Stochastic differential equations and applications, Woodhead Publishing, Cambridge, 2007.

[18] L. Rosier: Homogeneous Lyapunov function for homogeneous continuous vector field, Systems \& Control Letters, vol. 19, no. 6, pp. 467-473, 1992. 